\title{
The static tetraquark and pentaquark potentials
}

\author{
C. Alexandrou and G. Koutsou \\ Department of Physics, University of Cyprus, CY-1678 Nicosia, Cyprus
}

(Dated: July 6, 2018)

\begin{abstract}
We evaluate the static $q q \bar{q} \bar{q}$ and $q q q q \bar{q}$ potentials in the quenched theory at $\beta=5.8$ and $\beta=6.0$ on a lattice of size $16^{3} \times 32$. We compare the static potentials to the sum of two meson potentials for the tetraquark system and to the sum of the baryonic and mesonic potentials for the pentaquark state, as well as, with the confining potential obtained in the strong coupling expansion.

PACS numbers: 11.15.Ha, 12.38.Gc, 12.38.Aw, 12.38.-t, 14.70.Dj
\end{abstract}

\section{INTRODUCTION}

A large amount of effort has been devoted recently to experimental searches [1] for the identification of the $\Theta^{+}$, an exotic baryon state with an unusually narrow width, which was predicted theoretically in the chiral soliton model [2]. Several studies in lattice QCD have looked for such a state in order to determine its mass and parity but no consensus has been reached yet with some groups reporting a bound state with mass close to the experimental value [3, 4] and others the KN scattering state [5]. The possible existence of such a state has raised interesting questions about its structure in connection to its narrow width. A number of phenomenological models have been put forward to explain its stability such as special flux tube configurations [6, 7] and diquark formation 8]. Another exotic that might exist and has been proposed in the past [9] is a bound state of two quarks and two antiquarks. In this work we calculate the static $q q \bar{q} \bar{q}$ and $q q q q \bar{q}$ potentials. The study of the pentaquark potential is particularly important for our understanding of the underlying structure of the pentaquark bound states that have been detected in various experiments [1, 10].

To evaluate the static tetraquark and pentaquark potentials we construct the Wilson loops for the $q q \bar{q} \bar{q}$ and $q q q q \bar{q}$ systems. In the strong coupling approximation minimization of the energy of a system of two quarks and two antiquarks requires that the two quarks and the two antiquarks are connected by the minimal length flux tube. The flux tubes from each of the quarks and the antiquarks can meet at one or at two points. For the geometries considered in this work the length of the flux tube with two Steiner points is always smaller than the configuration with the flux tubes meeting at a point and therefore it corresponds to the minimal flux tube length. If the minimal flux tube length is denoted by $L_{\min }$ then the confining potential in the strong coupling approximation is $\sigma L_{\text {min }}$ where for $\sigma$ we take the string tension extracted from the quark-antiquark potential. Besides comparing the static tetraquark potential to the one extracted in the strong coupling approximation we also compare it to the sum of two meson potentials. Similarly the pentaquark potential is compared to the potential extracted in the strong coupling expansion and to the sum of the baryonic and mesonic potentials. Minimization of the energy of a pentaquark system with the condition that the flux tubes connecting the quarks meet at three points gives, as minimal length, the flux tube configuration where the three flux tube junctions are Steiner points. We do not consider here geometries resulting in the diamond flux tube arrangement [7] for which the flux tubes from the four quarks meet at a single point where the antiquark is located. Obtaining results for both the tetraquark and pentaquark potentials enables us to look for differences in their behaviour which can reflect different structures in the tetraquark and pentaquark systems. Such information may lead to important insight in our understanding of the structure of the $\Theta^{+}$.

\section{WILSON LOOPS}

The SU(3) Wilson loop for the tetraquark system is constructed by creating a gauge invariant four quark state at time $t=0$, which is annihilated at a later time $t$ as shown in Fig. 1. The two quarks are combined into a colour $\overline{3}$ and the two antiquarks into a colour 3 representation of $\mathrm{SU}(3)$. The expression for the tetraquark Wilson loop is given by [4]

$$
\begin{gathered}
W_{4 q}=\frac{1}{12} \epsilon^{a b c} \epsilon^{d e f} \epsilon^{a^{\prime} b^{\prime} c^{\prime}} \epsilon^{d^{\prime} e^{\prime} f^{\prime}} U\left(\mathbf{x}, \mathbf{x}^{\prime}, 1\right)^{a a^{\prime}} U\left(\mathbf{x}, \mathbf{x}^{\prime}, 2\right)^{b b^{\prime}} \\
U_{G}(\mathbf{x}, \mathbf{y})^{c f} U\left(\mathbf{y}^{\prime}, \mathbf{y}, 3\right)^{d^{\prime} d} U\left(\mathbf{y}^{\prime}, \mathbf{y}, 4\right)^{e^{\prime} e} U_{G^{\prime}}\left(\mathbf{y}^{\prime}, \mathbf{x}^{\prime}\right)^{f^{\prime} c^{\prime}}(1)
\end{gathered}
$$

where the two quark lines are created at $\mathbf{x}$ and the two antiquark lines at $\mathbf{y}$ at time $t=0$ and annihilated at $\mathbf{x}^{\prime}$ and $\mathbf{y}^{\prime}$ at time $t$ respectively. The staples $U\left(\mathbf{x}, \mathbf{x}^{\prime}, k\right)$ involved in the definition of the Wilson loop are given by

$$
U\left(\mathbf{x}, \mathbf{x}^{\prime}, k\right)=P \exp \left[i g \int_{\Gamma_{k}} d z^{\mu} A_{\mu}(z)\right]
$$

where $P$ is the path ordered operator and $\Gamma_{k}$ denotes the path from $\mathbf{x}$ to $\mathbf{x}^{\prime}$ for quark line $k$ as shown in Fig. 1] The baryonic junction at $\mathbf{x}$ is joined to the anti-baryonic junction at $\mathbf{y}$ by

$$
U_{G}(\mathbf{x}, \mathbf{y})=P \exp \left[i g \int_{G} d \mathbf{z} \cdot \mathbf{A}(z)\right]
$$

at $t=0 . U_{G^{\prime}}\left(\mathbf{y}^{\prime}, \mathbf{x}^{\prime}\right)$ is the corresponding arrow joining the anti-baryonic and baryonic junctions at time $t$. 


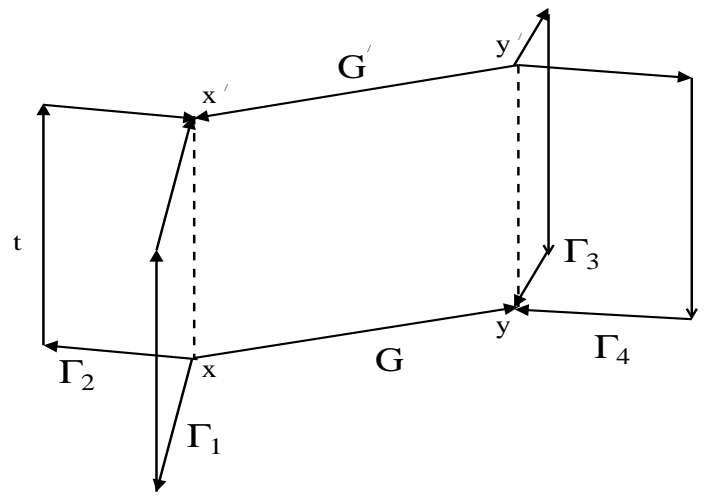

FIG. 1: The Wilson loop for the $q q \bar{q} \bar{q}$ system.

The tetraquark potential is then extracted in the standard way from the long time behaviour of the Wilson loop:

$$
V_{4 q}=-\lim _{t \rightarrow \infty} \frac{1}{t} \ln \left\langle W_{4 q}\right\rangle
$$

The pentaquark Wilson loop is constructed in a similar way: The gauge invariant state evolves now two baryonic and one anti-baryonic junction. The four quarks are grouped into two diquarks each in a colour $\overline{3}$ representation of $\mathrm{SU}(3)$ and the two diquarks are then combined into a colour singlet with the remaining antiquark. The Wilson loop is shown in Fig. [2 and it is given by [4]

$$
\begin{gathered}
W_{5 q}=\frac{1}{24}\left[\epsilon^{a b c} \epsilon^{a^{\prime} b^{\prime} c^{\prime}} U\left(\mathbf{x}, \mathbf{x}^{\prime}, 1\right)^{a a^{\prime}} U\left(\mathbf{x}, \mathbf{x}^{\prime}, 2\right)^{b b^{\prime}}\right] \\
{\left[\epsilon^{d e f} \epsilon^{d^{\prime} e^{\prime} f^{\prime}} U\left(\mathbf{z}, \mathbf{z}^{\prime}, 3\right)^{d d^{\prime}} U\left(\mathbf{z}, \mathbf{z}^{\prime}, 4\right)^{e e^{\prime}}\right] U\left(\mathbf{y}^{\prime}, \mathbf{y}, 5\right)^{j^{\prime} j}} \\
\epsilon^{g h j} \epsilon^{g^{\prime} h^{\prime} j^{\prime}} U_{G_{1}}(\mathbf{x}, \mathbf{y})^{c g} U_{G_{2}}(\mathbf{z}, \mathbf{y})^{f h} \\
U_{G_{1}^{\prime}}\left(\mathbf{y}^{\prime}, \mathbf{x}^{\prime}\right)^{g^{\prime} c^{\prime}} U_{G_{2}^{\prime}}\left(\mathbf{y}^{\prime}, \mathbf{z}^{\prime}\right)^{h^{\prime} f^{\prime}}
\end{gathered}
$$

using the same notation as that of Eq. (1).

\section{LATTICE TECHNIQUES}

A number of improvements are needed in order to reduce the noise in the measurement of the Wilson loops. Since the tetraquark and pentaquark potentials are larger than the baryonic potential they are harder to compute and noise reduction methods are essential. We describe briefly the techniques that we use in order to reduce noise and extract reliably the ground state:

1. We use the multi-hit procedure [11] replacing the temporal links by their average value

$$
U_{4}(x) \rightarrow \bar{U}_{4}(x)=\frac{\int d U U_{4}(n) e^{\beta S_{4}(U)}}{\int d U e^{\beta S_{4}(U)}}
$$

with $S_{4}(U)=\frac{1}{N} \operatorname{Tr}\left(U_{4}(n) F^{\dagger}(n)\right)$ and $F(n)$ the staple attached to the time link that is being integrated over. The

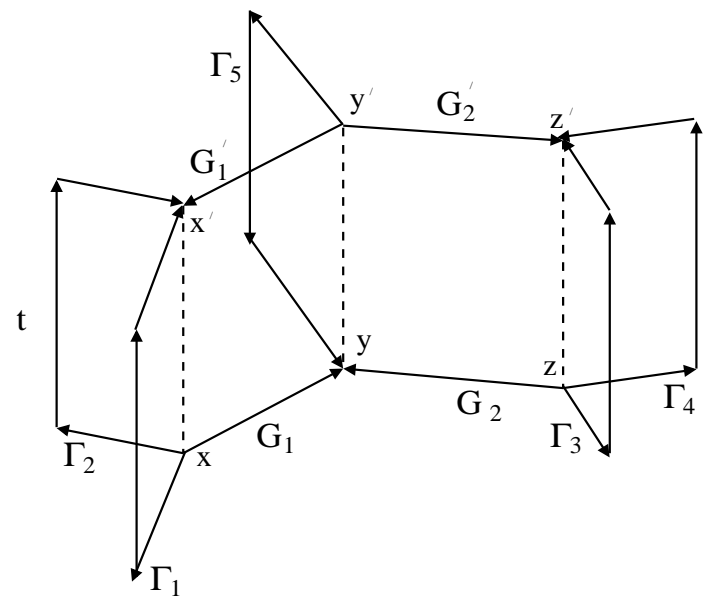

FIG. 2: The Wilson loop for the $q q q q \bar{q}$ system.

integration over the links in Eq. (6) is carried out analytically [12]. It has been shown in $\mathrm{SU}(2)$ 13] that replacing the time links by their average value in this fashion reduces the error on large Wilson loops of the order of ten. The factor found in ref. [13] is $x^{2 t} \sim 0.889^{2 t}$ where $t$ is the time extent of the Wilson loop. For the tetraquark and pentaquark Wilson loops the reduction factor will be $x^{4 t}$ and $x^{5 t}$ respectively giving an even larger noise reduction for the large loops.

2. To maximize the overlap of the trial state with the four or five quark ground state we use APE smearing of the spatial links 14]. Each spatial link is replaced by a fat link by acting on it with the smearing operator $\mathcal{S}$ defined by

$\mathcal{S} U_{j}(x)=\mathcal{P}\left(U_{j}(x)+\alpha \sum_{k \neq j}\left[U_{k}(x) U_{j}(x+a \hat{k}) U_{k}^{\dagger}(x+a \hat{j})\right]\right)$,

where $\mathcal{P}$ denotes projection onto $\mathrm{SU}(3)$. This is iterated $n$ times. We consider $M$ different levels of smearing and construct an $M \times M$ correlation matrix of Wilson loops. We take $\alpha=1 / 2, M=3$ and successive number of smearings $n_{1}=0, n_{2}=15$ and $n_{3}=30$.

The correlation matrices $C(t)$ for the various Wilson loops are analyzed using a variational method [15]. We analyze the results in two different ways:

In the first variant, which we refer to as variational method 1, we solve the generalized eigenvalue problem

$$
C(t) v_{k}(t)=\lambda_{k}(t) C\left(t_{0}\right) v_{k}(t) \quad k=1, \ldots, M \quad,
$$

taking $t_{0} / a=1$ and extract the potential levels via

$$
a V_{k}=\lim _{t \rightarrow \infty}-\left[\ln \left(\frac{\lambda_{k}(t)}{\lambda_{k}(t-1)}\right)\right]
$$

by fitting to a constant in the range where $a V_{k}$ becomes time independent (plateau region).

In the second variant that we call variational method 2 we first solve the eigenvalue equation

$$
C\left(t_{0}\right) v_{k}\left(t_{0}\right)=\lambda_{k}\left(t_{0}\right) v_{k}\left(t_{0}\right)
$$


and project the Wilson correlation matrices to the space corresponding to the $\mathrm{N}$ largest eigenvalues

$$
C_{i j}^{N}(t)=\left(v_{i}\left(t_{0}\right), C(t) v_{j}\left(t_{0}\right)\right)
$$

with $N \leq M$. We then solve the generalized eigenvalue equation

$$
C^{N}(t) v_{k}(t)=\lambda_{k}(t) C^{N}\left(t_{0}\right) v_{k}(t)
$$

in the truncated space.

In addition the potential is extracted by considering the Wilson loop with the largest number of smearings and fit to the ratio

$$
V=\lim _{t \rightarrow \infty}-\ln [W(t) / W(t-1)]
$$

in the plateau region.

The noise reduction techniques described above were shown to yield accurate enough results for the baryonic potential for inter-quark distances of $\mathcal{O}(1.5) \mathrm{fm}$ [16].

\section{RESULTS}

All the computations were carried out on a lattice of size $16^{3} \times 32$ at $\beta$ values 5.8 and 6.0 using 200 and 220 configurations respectively available at the NERSC archive [17].

We compute the static $q \bar{q}$ potential on the same configurations used for the evaluation of the tetraquark and pentaquark potentials. Fitting the $q \bar{q}$ potential to the Cornell Ansatz

$$
V_{q \bar{q}}(r)=V_{0}-\frac{\alpha}{r}+\sigma r
$$

we extract the parameters $V_{0}, \alpha$ and string tension $\sigma$. We give the values obtained in Table [ Using the value of $a^{2} \sigma$ given in Table $\Pi$ and the physical value of $\sqrt{\sigma}=440 \mathrm{MeV}$ known from Regge theory enables us to fix the lattice spacing $a$. We obtain $a=0.10 \mathrm{fm}$ at $\beta=6.0$ and $a=0.15 \mathrm{fm}$ at $\beta=5.8$. Since in this work we compare the tetraquark potential to the sum of two meson potentials and the pentaquark potential to the sum of the corresponding mesonic and baryonic potentials we need to compute all the potentials with the quarks at the same locations. For the mesonic potential needed in the comparison of the tetraquark system we use lattice data avoiding any parametrizations. For the baryonic potential needed in the comparison of the pentaquark potential we do not have lattice data and therefore we need a parametrization. Two possible Ansätze have been discussed in the literature for the baryonic potential, the Y-Ansatz [18] and the sum of twobody potentials or $\Delta$-Ansatz. The latter was derived using center vortices 19 but with an erroneous assumption, which after correction was shown to support the Y-Ansatz 20]. Nevertheless within lattice QCD one can check the two Ansätze and determine whether the confining potential is closer to a sum of two-body potentials
TABLE I: The parameters of the static $q \bar{q}$ potential in lattice units at $\beta=5.8$ and $\beta=6.0$.

\begin{tabular}{|c|c|c|c|}
\hline$\beta$ & $a V_{0}$ & $\alpha$ & $a^{2} \sigma$ \\
\hline \hline 6.0 & $0.637(5)$ & $0.255(5)$ & $0.050(1)$ \\
\hline 5.8 & $0.636(11)$ & $0.248(11)$ & $0.105(2)$ \\
\hline
\end{tabular}

or to the Y-Ansatz as a function of the distance between the quarks. The results of the two Ansätze differ at the most by $15 \%$ and have been compared to lattice results in refs. 16, 21, 22, 23, 24, 25]. The general consensus from these studies is that at large distances the baryonic potential approaches the Y-Ansatz. Therefore here it suffices to use the Y-Ansatz to parametrize the baryonic potential. As in ref. [16] we use the values for $V_{0}, \alpha$ and $\sigma$ extracted from fitting the $q \bar{q}$ potential via Eq. (14) and given in Table I This means that there are no adjustable parameters in the comparison of the pentaquark potential to the sum of the mesonic and baryonic potential. In addition both the tetraquark and pentaquark potentials are compared to the potential derived in the strong coupling expansion. We use the parametrization given by

$$
\left(\begin{array}{c}
V_{\min }^{4 q} \\
V_{\min }^{5 q}
\end{array}\right)=\left(\begin{array}{c}
2 \\
5 / 2
\end{array}\right) V_{0}-n_{q} \sum_{i>j} \frac{\alpha}{\left|\mathbf{r}_{i}-\mathbf{r}_{j}\right|}+\sigma\left(\begin{array}{c}
L_{\min }^{4 q} \\
L_{\min }^{5 q}
\end{array}\right),
$$

where again for $V_{0}, \alpha$ and $\sigma$ we use the values given in Table प The factor $n_{q}$ in front of the Coulomb term is one when a quark interacts with an antiquark and one half when the interaction is between quarks or between antiquarks as obtained from the one-gluon exchange approximation.

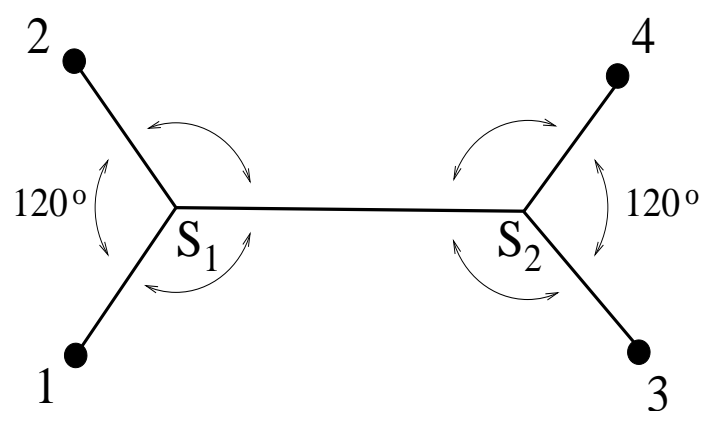

FIG. 3: The tetraquark minimal length. The angles at the junctions are $120^{\circ}$.

For a general tetraquark configuration the minimal length having two Steiner points is shown in Fig. B and, in general, is non-planar. For the planar geometry considered in this work the minimal length can be easily computed and it is given by

$$
L_{\text {min }}^{4 q}=\min \left(R_{1}+\sqrt{3} R_{2}, R_{2}+\sqrt{3} R_{1}\right),
$$

where the distances $R_{1}$ and $R_{2}$ are defined in Fig. 4 For 


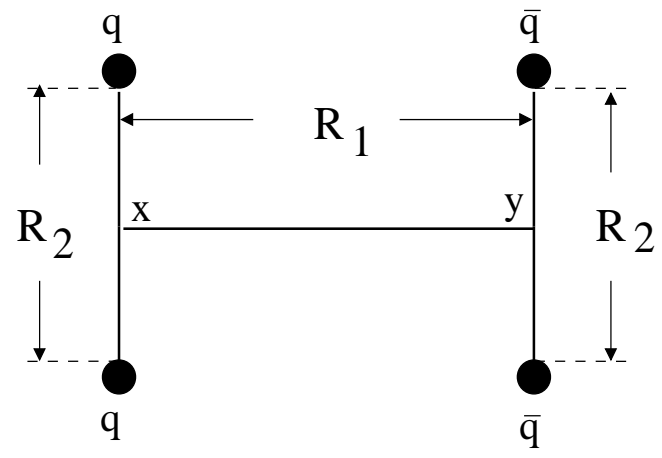

FIG. 4: The geometry used for the computation of the tetraquark static potential where we have taken the baryonic junction at the origin and the anti-baryonic junction at $\mathbf{y}=\left(R_{1}, 0,0\right)$. The quarks are located at positions $\mathbf{r}_{1}=\left(0, R_{2} / 2,0\right), \mathbf{r}_{2}=\left(0,-R_{2} / 2,0\right)$ and the antiquarks at $\mathbf{r}_{3}=\left(R_{1}, R_{2} / 2,0\right)$ and $\mathbf{r}_{4}=\left(R_{1},-R_{2} / 2,0\right)$.

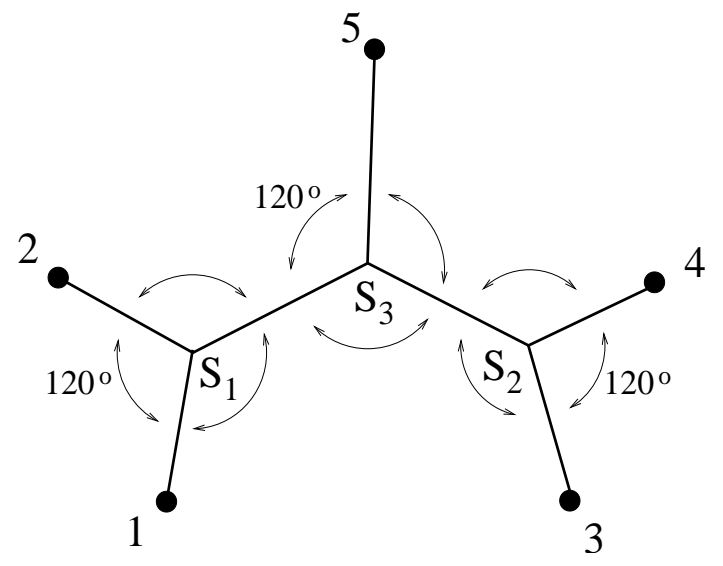

FIG. 5: The pentaquark minimal length. The angles at the junctions are all $120^{\circ}$.

a general pentaquark configuration the minimal length with three junctions is non planar and has the geometry shown in Fig. [5 with the three junctions being Steiner points. We compute these three Steiner points numerically as follows: We start by an initial guess for the Steiner point $S_{3}$. Having an initial guess for $S_{3}$ enables us to evaluate the two other Steiner points analytically in terms of $S_{3}$ following ref. [18]:

$$
\begin{aligned}
& \left|\mathbf{r}_{i}-\mathbf{r}_{S_{1}}\right|=\frac{C^{2}-r_{j k}^{2}}{B} \quad(i, j, k \quad \text { cyclic }) \\
& C^{2}=\frac{1}{2} \xi+\left(\frac{1}{3} \eta-\frac{1}{12} \xi^{2}\right)^{1 / 2} \quad B^{2}=3 C^{2}-\xi \\
& \xi=r_{12}^{2}+r_{23}^{2}+r_{31}^{2}
\end{aligned}
$$

We discuss first the results obtained for the tetraquark system. As already mentioned the geometry that we use

$$
\eta=r_{12}^{2} r_{23}^{2}+r_{23}^{2} r_{31}^{2}+r_{31}^{2} r_{12}^{2}
$$

where $r_{12}=\left|\mathbf{r}_{1}-\mathbf{r}_{2}\right|, r_{23}=\left|\mathbf{r}_{2}-\mathbf{r}_{S_{3}}\right|$, and $r_{31}=\left|\mathbf{r}_{S_{3}}-\mathbf{r}_{1}\right|$. The minimal length joining the three points $\mathbf{r}_{1}, \mathbf{r}_{2}$ and $\mathbf{r}_{S_{3}}$ is equal to $B$. If any interior angle of the triangle $\mathbf{r}_{1} \mathbf{r}_{2} \mathbf{r}_{\mathbf{S}_{3}}$ is greater than $120^{\circ}$ then the minimal length is given by $r_{12}+r_{23}+r_{31}-\max \left(r_{12}, r_{23}, r_{31}\right)$. This is taken

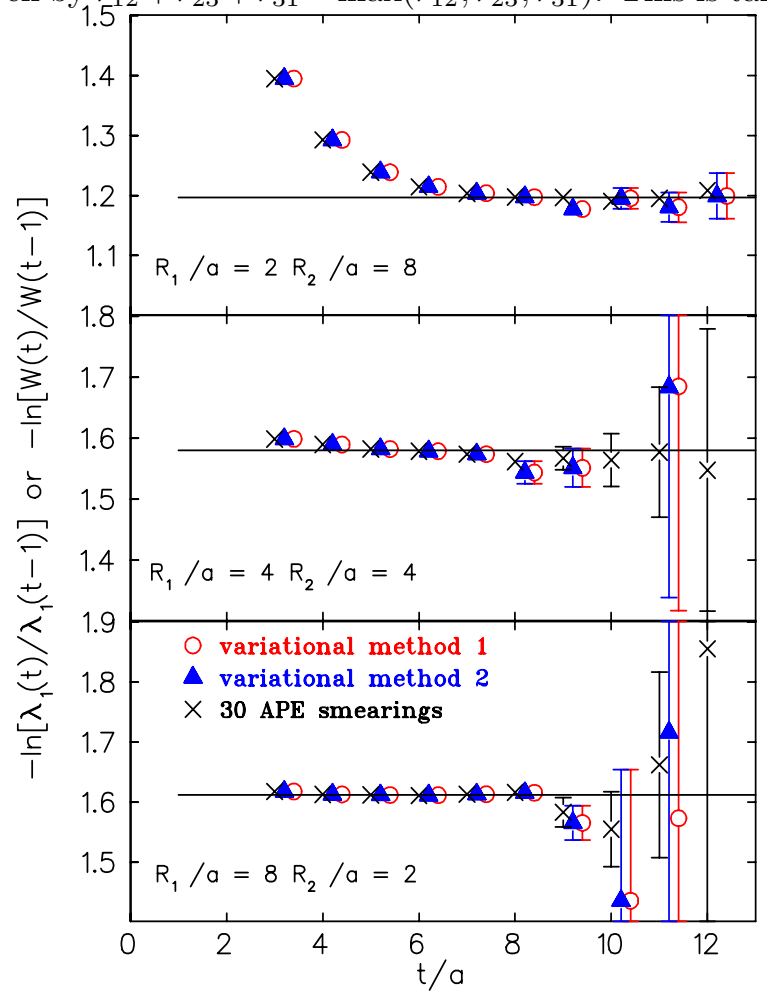

FIG. 6: Plateau values for the tetraquark potential. Top for the case where $R_{1} / a=2$ and $R_{2} / a=8$; middle for $R_{1} / a=4$ and $R_{2} / a=4$; bottom for $R_{1} / a=8$ and $R_{2} / a=2$. The data obtained using variational method 1 are denoted by the circles, using variational method 2 by the filled triangles and using Eq. (13) by the crosses. The circles and filled triangles are shifted horizontally for clarity.

into account in our analytic determination of the Steiner points. An analogous expression holds for $S_{2}$. Having a value for $S_{1}$ and $S_{2}$ and knowing $\mathbf{r}_{5}$ we can evaluate analytically the new value for $S_{3}$. This procedure is iterated until convergence is reached after a small number (of order 10) of iterations. We use this method to compute $L_{\text {min }}^{5 q}$ for the geometry studied in this work. This approach can also be applied to find the minimal length for a general tetraquark geometry.

for the computation of the tetraquark potential is planar and is shown in Fig. 4. When $R_{2}$ is small this configu- 
TABLE II: Determination of plateau values for the tetraquark potential at $\beta=6.0$. The first column gives the diquark distance, $R_{1}$, and for each of the internal diquark distances, $R_{2}$, we give, the the initial and final time $t_{i} / a$ and $t_{f} / a$ used in fitting the plateau, the value of the potential and $\tilde{\chi}^{2}=\chi^{2} /$ d.o.f. We denote with an asterisk the value that we choose. All quantities are given in lattice units.

\begin{tabular}{|c|c|c|c|c|c|c|c|c|c|c|c|c|c|c|c|c|}
\hline \multicolumn{17}{|c|}{ Tetraquark potential } \\
\hline$R_{1} / a$ & \multicolumn{4}{|c|}{$R_{2} / a=2$} & \multicolumn{4}{|c|}{$R_{2} / a=4$} & \multicolumn{4}{|c|}{$R_{2} / a=6$} & \multicolumn{4}{|c|}{$R_{2} / a=8$} \\
\hline \multirow{4}{*}{2} & $t_{i} / a$ & $t_{f} / a$ & $a V_{4 q}$ & $\tilde{\chi}^{2}$ & $t_{i} / a$ & $t_{f} / a$ & $a V_{4 q}$ & $\tilde{\chi}^{2}$ & $t_{i} / a$ & $t_{f} / a$ & $a V_{4 q}$ & $\tilde{\chi}^{2}$ & $t_{i} / a$ & $t_{f} / a$ & $a V_{4 q}$ & $\tilde{\chi}^{2}$ \\
\hline & 8 & 12 & $1.195(1)$ & 13 & 8 & 12 & $1.219(1)$ & 32 & 8 & 12 & $1.201(1)$ & 5.7 & 7 & 12 & $1.200(1)$ & 5.4 \\
\hline & 10 & 12 & $1.190(1)^{*}$ & 1.2 & 10 & 12 & $1.203(2)^{*}$ & 0.6 & 9 & 12 & $1.196(1)^{*}$ & 0.8 & 8 & 12 & $1.197(1)^{*}$ & 1.4 \\
\hline & 11 & 12 & $1.190(2)$ & 0.6 & 11 & 12 & $1.200(3)$ & 0.1 & 11 & 12 & $1.191(4)$ & 0.5 & 9 & 12 & $1.195(2)$ & 1.6 \\
\hline \multirow{3}{*}{3} & $J$ & 12 & $318(1)$ & 1.2 & 7 & 12 & $.450(2)$ & 3.7 & 6 & 12 & $1.473(2)$ & 16 & 7 & 12 & $1.428(4)$ & 2.4 \\
\hline & 6 & 12 & $1.318(1)^{*}$ & 0.8 & 8 & 12 & $1.441(4)^{*}$ & 1.2 & 8 & 12 & $1.435(6)^{*}$ & 0.8 & 8 & 12 & $1.409(7)^{*}$ & 0.2 \\
\hline & 8 & 12 & $1.317(1)$ & 0.9 & 9 & 12 & $1.430(7)$ & 0.1 & 10 & 12 & $1.435(24)$ & 0.4 & 9 & 12 & $1.407(15)$ & 0.3 \\
\hline \multirow{3}{*}{4} & 4 & 12 & $1.390(1)^{*}$ & 1.2 & 4 & 12 & $1.584(1)$ & 5.2 & 6 & 12 & $1.646(3)$ & 2.8 & 6 & 12 & $1.645(6)$ & 3.4 \\
\hline & 7 & 12 & $1.390(1)$ & 1.6 & 6 & 12 & $1.577(2)^{*}$ & 0.8 & 8 & 12 & $1.605(16)^{*}$ & 1.4 & 6 & 10 & $1.646(6)^{*}$ & 0.7 \\
\hline & 10 & 12 & $1.386(6)$ & 2.6 & 9 & 12 & $1.567(20)$ & 0.1 & 10 & 12 & $1.560(99)$ & 0.7 & 7 & 10 & $1.629(13)$ & 0.3 \\
\hline \multirow{3}{*}{5} & 4 & 12 & $1.450(1)^{*}$ & 0.6 & 5 & 10 & $1.651(2)^{*}$ & 1.1 & 5 & 12 & $1.780(3)$ & 1.4 & 5 & 12 & $1.861(6)$ & 2.4 \\
\hline & 7 & 12 & $1.451(2)$ & 0.5 & 6 & 10 & $1.648(3)$ & 0.7 & 6 & 12 & $1.767(7)^{*}$ & 0.5 & 6 & 12 & $1.823(15)^{*}$ & 0.2 \\
\hline & 10 & 12 & $1.447(11)$ & 0.8 & 7 & 10 & $1.642(6)$ & 0.4 & 8 & 12 & $1.725(42)$ & 0.2 & 7 & 12 & $1.826(41)$ & 0.2 \\
\hline \multirow{3}{*}{6} & 3 & 12 & $1.508(1)^{*}$ & 0.8 & 4 & 12 & $1.716(2)^{*}$ & 0.6 & 4 & 12 & $1.881(2)$ & 7.4 & 4 & 11 & $2.010(4)$ & 4.3 \\
\hline & 7 & 12 & $1.507(3)$ & 0.1 & 7 & 12 & $1.717(10)$ & 0.6 & 6 & 12 & $1.846(10)^{*}$ & 1.4 & 6 & 11 & $1.948(25)^{*}$ & 0.8 \\
\hline & 10 & 12 & $1.503(21)$ & 0.1 & 9 & 12 & $1.656(64)$ & 0.2 & 10 & 12 & $1.278(808)$ & 0.1 & 7 & 11 & $2.043(86)$ & 0.7 \\
\hline \multirow{3}{*}{7} & 4 & 12 & $1.560(2)^{*}$ & 0.5 & 3 & 7 & $1.773(2)$ & 4.0 & 4 & 9 & $1.933(4)$ & 2.2 & 4 & 12 & $2.088(6)$ & 2.0 \\
\hline & 7 & 12 & $1.560(4)$ & 0.7 & 5 & 7 & $1.766(3)^{*}$ & 0.7 & 6 & 9 & $1.918(14)^{*}$ & 0.6 & 6 & 12 & $2.015(36)^{*}$ & 0.5 \\
\hline & 10 & 12 & $1.563(36)$ & 1.4 & 5 & 9 & $1.765(3)$ & 5.7 & 7 & 9 & $1.909(41)$ & 0.9 & 7 & 12 & $1.906(107)$ & 0.4 \\
\hline \multirow{3}{*}{8} & 4 & 12 & $1.612(2)^{*}$ & 0.4 & 6 & 12 & $1.804(7)^{*}$ & 1.1 & 4 & 10 & $1.987(4)$ & 1.7 & 6 & 12 & $2.080(53)$ & 1.9 \\
\hline & 7 & 12 & $1.612(6)$ & 0.6 & 7 & 12 & $1.778(19)$ & 0.9 & 6 & 10 & $1.963(19)^{*}$ & 0.2 & 6 & 8 & $2.097(54)^{*}$ & 0.9 \\
\hline & 10 & 12 & $1.574(67)$ & 0.4 & 9 & 12 & $1.813(144)$ & 0.8 & 8 & 10 & $1.941(20)$ & 0.3 & 7 & 8 & 1.916(109) & 0.1 \\
\hline
\end{tabular}

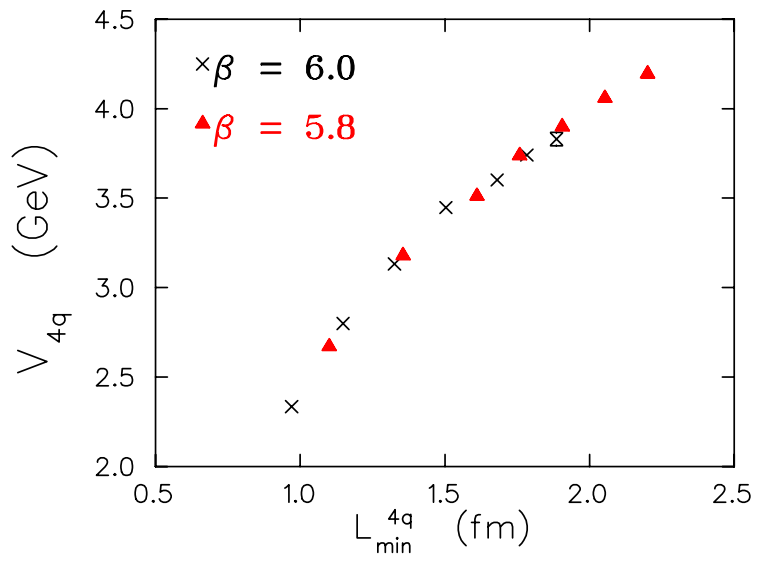

FIG. 7: The tetraquark static potential at $\beta=6.0$ (crosses) and $\beta=5.8$ (filled triangles) for $R_{2}=0.6 \mathrm{fm}$ as a function of the minimal length in physical units. We have applied a constant shift to the data at $\beta=5.8$. The jackknife errors are smaller than the size of the symbols.

ration allows diquark formation. We take the distance between the two quarks and the two antiquarks, $R_{2}$, to be equal. We will refer to $R_{2}$ as the internal diquark distance. The potential is computed as a function of the distance between the two diquarks, $R_{1}$, and the internal diquark distance $R_{2}$. In Fig. 6 we show the plateaus for representative values of the distances $R_{1}$ and $R_{2}$. On these figures we display the values extracted using the two variational methods as well as the one extracted from the ratio of Wilson loops with the largest number of APE smearings as given in Eq. (13). As can be seen from these figures the two variational methods yield consistent results of similar quality. The ratio of the Wilson loops on the other hand yields better plateaus. This is true for all the distances that we have performed this analysis and we will therefore use this method to extract the potential. We have always checked that the variational analysis gave results consistent with those extracted using Eq. (13). The errors shown on all our figures are jackknife errors.

We check scaling by comparing results at $\beta=5.8$ and $\beta=6.0$. In Fig. [7 we show the static tetraquark potential for the same physical internal diquark distance $R_{2}$ as a function of the minimal length. We apply a constant shift to the data at $\beta=5.8$ given by twice the difference in the constants $V_{0}$ at the two $\beta$ values. As can be seen the results, to a good approximation, fall on a universal line showing reasonable scaling behaviour. As in all our figures the errors shown on this figure are the 


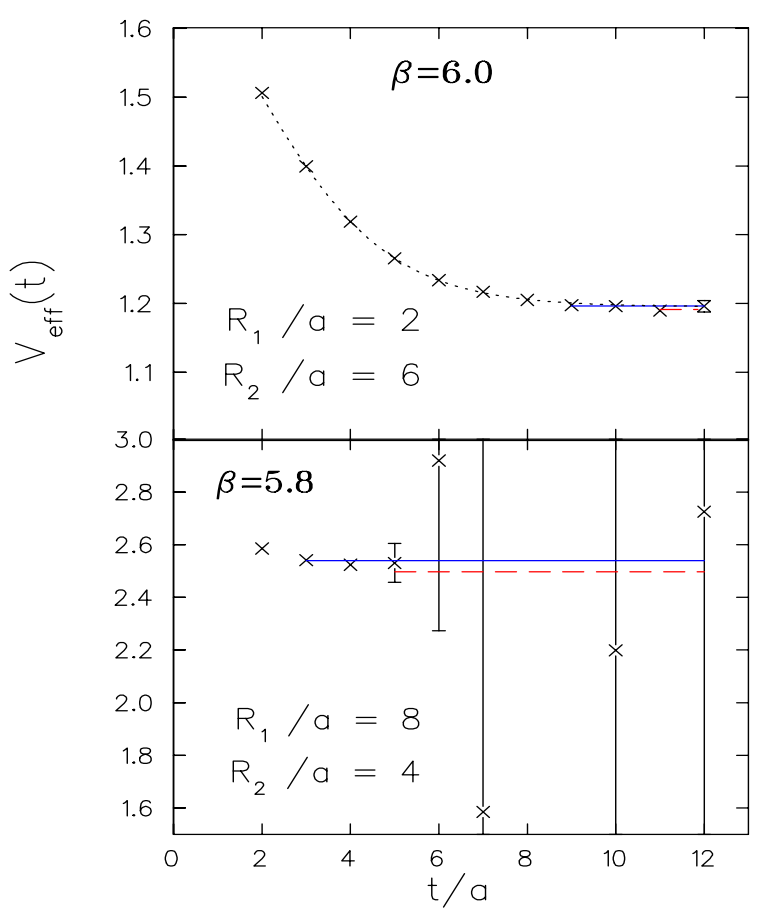

FIG. 8: The ratio $-\log \frac{W(t)}{W(t-1)}$ for the tetraquark static potential versus time in lattice units. Upper graph for $R_{1}=0.2 \mathrm{fm}$ and $R_{2}=0.6 \mathrm{fm}$ at $\beta=6.0$. Lower graph for $R_{1}=1.2 \mathrm{fm}$ and $R_{2} / a=0.6 \mathrm{fm}$ at $\beta=5.8$. Horizontal lines show the value obtained from fitting the lattice data to a constant with the solid line showing the value that we choose. The initial time used in the fit is shown by the position where the line begins. The dotted curve shows the result of fitting to Eq. 18

statistical errors obtained by jackknife analysis. To understand why the statistical errors are small even at the larger distances we plot in Fig. 8 for two representative cases, the ratio $V_{\text {eff }}(t)=-\log \frac{W(t)}{W(t-1)}$, which for large $t$ gives the ground state potential $V_{4 q}$ we are interested in. The two cases that we consider are those that have the smallest and largest values of $L_{\min }$ in Fig. 7 The first corresponds to $R_{1}=0.2 \mathrm{fm}$ at $\beta=6.0$ and the second to $R_{1}=1.2 \mathrm{fm}$ at $\beta=5.8$ with $R_{2}=0.6 \mathrm{fm}$ in both cases. To fit to a constant we must search for a plateau of $V_{\text {eff }}(t)$ and make sure that changing the initial fitting range does not produce a value outside the statistical errors. In our analysis we take the plateau value that gives, for the earliest initial time range, $\tilde{\chi}^{2} \equiv \chi^{2} /$ d.o.f. $\lesssim 1$ since this criterion ensures a good fit and gives a result with the smallest statistical error. When $R_{1}=0.2 \mathrm{fm}$ the results are very accurate and clearly show contributions from excited states with $V_{\text {eff }}(t)$ showing a plateau only for $t / a>7$. Fitting within the plateau range we extract the values given in Table II where we check consistency by changing the initial fit range. Using our criterion we choose the plateau value with $\tilde{\chi}^{2}=0.8$ denoted by the asterisk in Table III At these small distances the accuracy of the data allows, in addition, a fit that includes the first

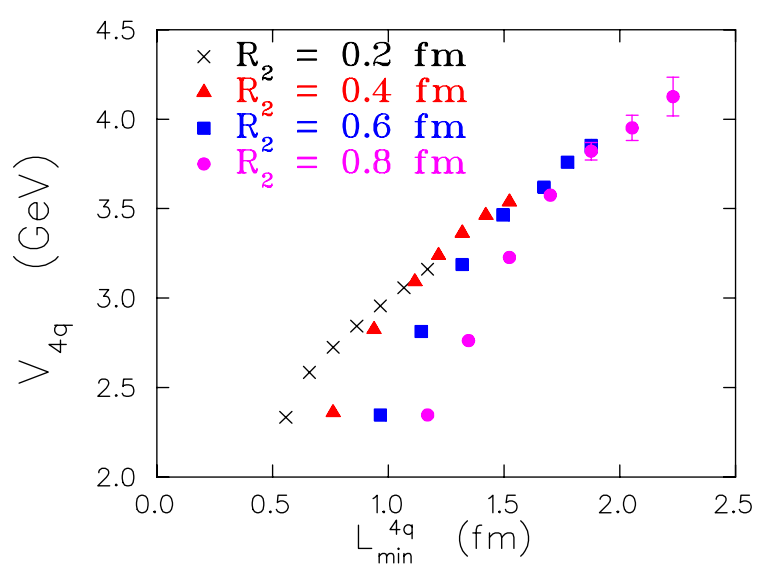

FIG. 9: The tetraquark static potential at $\beta=6.0$ versus the minimal length. Data obtained for $R_{2}=0.2,0.4,0.6$ and $0.8 \mathrm{fm}$ are denoted with crosses, filled triangles, filled squares and filled circles respectively.

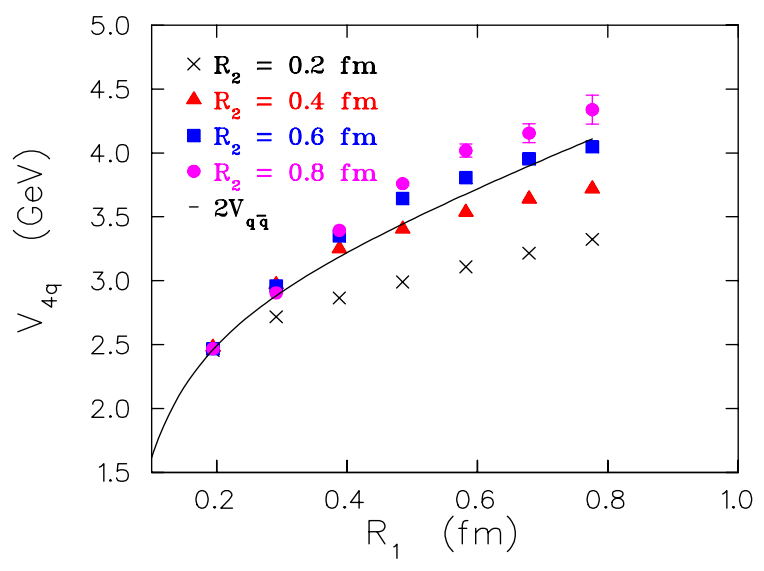

FIG. 10: The tetraquark static potential versus $R_{1}$ for $R_{2}=0.2 \mathrm{fm}$ (crosses), $0.4 \mathrm{fm}$ (filled triangles), $0.6 \mathrm{fm}$ (filled squares) and $0.8 \mathrm{fm}$ (filled circles). The solid line is twice the $q \bar{q}$ potential.

excited state. This enables us to extend the time range of the fit and check that including the first excited state reproduces the plateau value obtained for the ground state from fitting to a constant at larger times. In this case the time-dependence of $V_{\text {eff }}(t)$ is given by

$$
V_{\text {eff }}(t)=V_{4 q}-\log \left[\frac{1+c e^{-d V t}}{1+c e^{-d V(t-1)}}\right]
$$

where $d V$ is the gap between the ground state and the first excited state. Fitting from $t_{i} / a=3$ we obtain $a V_{4 q}=1.195(1)$, which agrees with the value extracted by fitting to a constant within the range of the plateau. When we consider the second case at the largest value of $L_{\text {min }}$ in Fig. [7]we see from Fig. [8 that $V_{\text {eff }}(t)$ has very large errors when $t / a>5$. This means that the first accurate points used in the fit will determine the value and error of the extracted constant and that checking for excited state contributions is no longer possible. In this partic- 


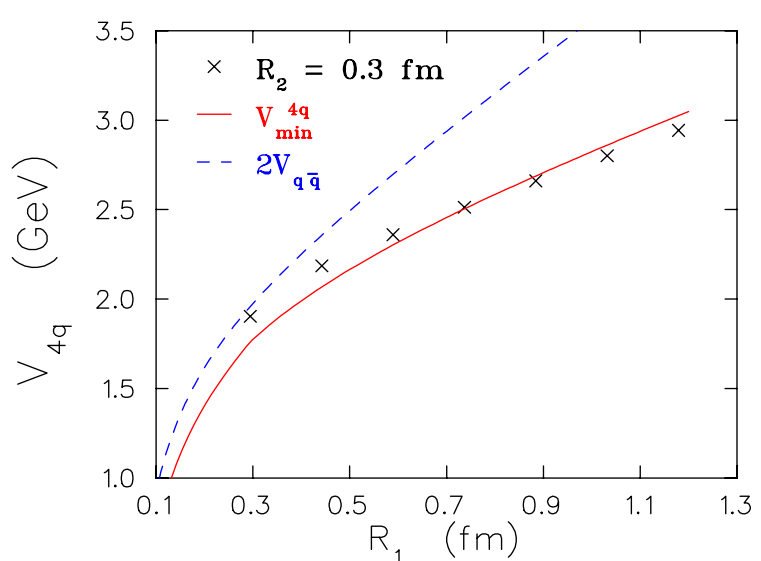

FIG. 11: The tetraquark static potential at $\beta=5.8$ for $R_{2}=0.3 \mathrm{fm}$ compared with $V_{\min }^{4 q}$ (solid line) and with $2 V_{q \bar{q}}\left(R_{1}\right)$ (dashed line).

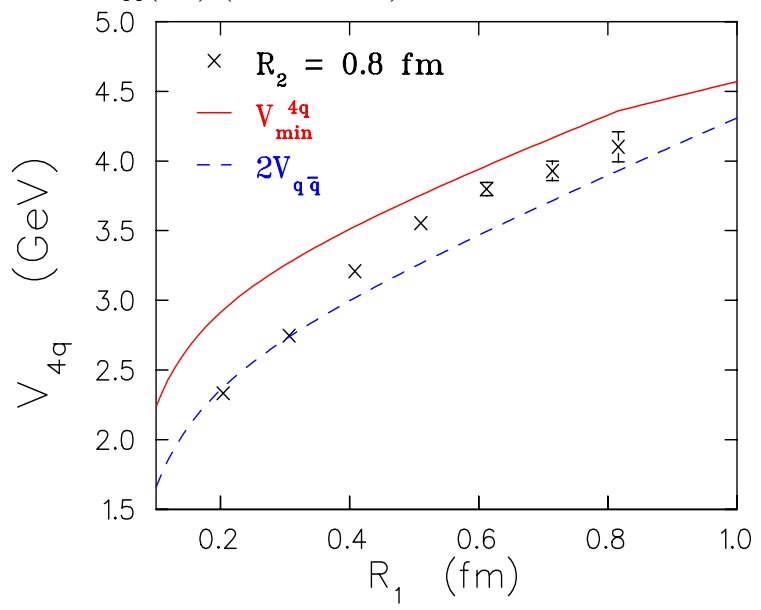

FIG. 12: The tetraquark static potential at $\beta=6.0$ for $R_{2}=0.8 \mathrm{fm}$ compared with $V_{\min }^{4 q}$ (solid line) and with $2 V_{q \bar{q}}\left(R_{1}\right)$ (dashed line).

ular example fitting to a constant from $t_{i} / a=3$ gives $\tilde{\chi}^{2}<1.0$ with an error on the extracted value which is small since it is basically determined from the two first accurate points. Changing the initial fit range to $t_{i} / a=5$ lowers the plateau value as shown in the figure but carries an error similar to that of the data point at $t / a=5$ making it consistent with the value extracted from the fit that used $t_{i} / a=3$. Within our fit criterion we choose the value obtained from the fit with $t_{i} / a=3$, which carries a small error explaining the small error of the data point at the largest value $L_{\min }$ in Fig. 7 We note that for these large interquark distances changing the final time range will not affect the value and error of the extracted potentials since the Wilson loops become very noisy at the larger times. In this particular example whether we take the upper fit range to be $t_{f} / a=7$ or $t_{f} / a=12$ does not affect the value and error resulting from the fit. As can be seen from Tableஹin general we choose the larger available value of $t_{f} / a$ but for the large distances we reduce the upper fit range when the data points become

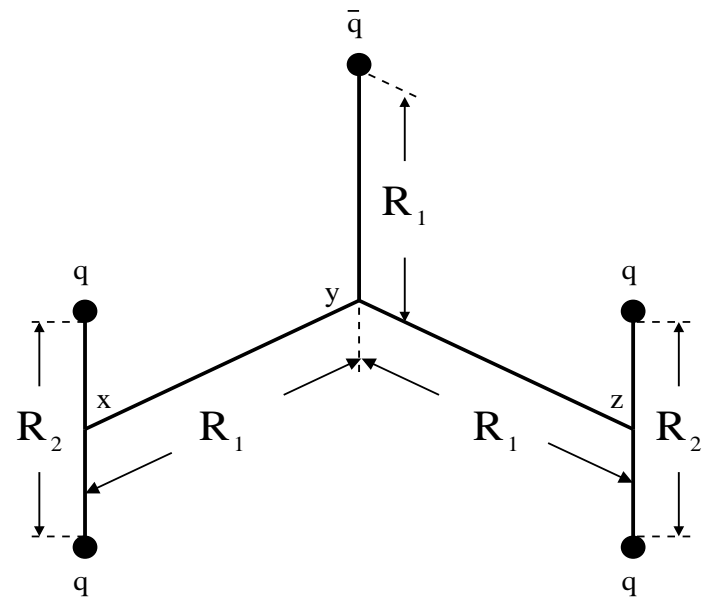

FIG. 13: The geometry used for the computation of the pentaquark static potential where we have taken the baryonic junctions at $\mathbf{x}=\left(R_{1}, 0,0\right)$ and at $\mathbf{z}=\left(0, R_{1}, 0\right)$ and the antibaryonic junction at $\mathbf{y}=(0,0,0)$. The quarks are located at positions $\mathbf{r}_{1}=\left(R_{1}, 0, R_{2} / 2\right), \mathbf{r}_{2}=\left(R_{1}, 0,-R_{2} / 2\right), \mathbf{r}_{3}=$ $\left(0, R_{1}, R_{2} / 2\right)$ and $\mathbf{r}_{4}=\left(0, R_{1},-R_{2} / 2\right)$ and the antiquark at $\mathbf{r}_{5}=\left(0,0, R_{1}\right)$.

too noisy. Since our accurate data points are limited to smaller times the plateau values that we extract may have excited state contributions, which can not be estimated since small shifts in the data points are not visible due to their large errors. However the fact that the data at the larger values of $L_{\text {min }}$ fall on the same curve as the better determined points is an indication that this error is reasonably small. In Table II we give the dependence of the plateau values on the fitting range and our choice of the value plotted in the figures. The table is done for $\beta=6.0$ since this is the case that we discuss in more detail in the manuscript. The analysis at $\beta=5.8$ is carried out in the same way.

In Fig. 9 we show the results for the tetraquark potential at $\beta=6.0$ as a function of the minimal length. As can be seen we have four different sets of curves for each internal diquark separation $R_{2}=0.2,0.4,0.6$ and $0.8 \mathrm{fm}$. When the distance between the diquarks becomes greater than the internal diquark distance the four sets of curves converge to the same line. This means that for $R_{1}>R_{2}$ the tetraquark potential becomes approximately only a function of $L_{\min }^{4 q}$. On the other hand when $R_{1} \leq R_{2}$ and for $R_{1} \lesssim 0.4 \mathrm{fm}$ the potential is independent of $R_{2}$. This behaviour is better seen in Fig. 10 where we show the tetraquark potential as a function of $R_{1}$ for the four different values of the internal diquark distance $R_{2}$ : When $R_{1}=0.2 \mathrm{fm}$ the potentials coincide for all four different values of $R_{2}$. When $R_{1}=0.3 \mathrm{fm}$ and, to a good approximation, also when $R_{1}=0.4 \mathrm{fm}$ the potentials coincide only for $R_{2}=0.4,0.6,0.8 \mathrm{fm}$. In fact, for $R_{1}=0.2 \mathrm{fm}$ and $0.3 \mathrm{fm}$ and $R_{2} \geq R_{1}$, the tetraquark potential is given by the sum of the two meson potentials as can be seen by the agreement of these results with the line on the figure showing $2 V_{q \bar{q}}\left(R_{1}\right)$. This means that for these 


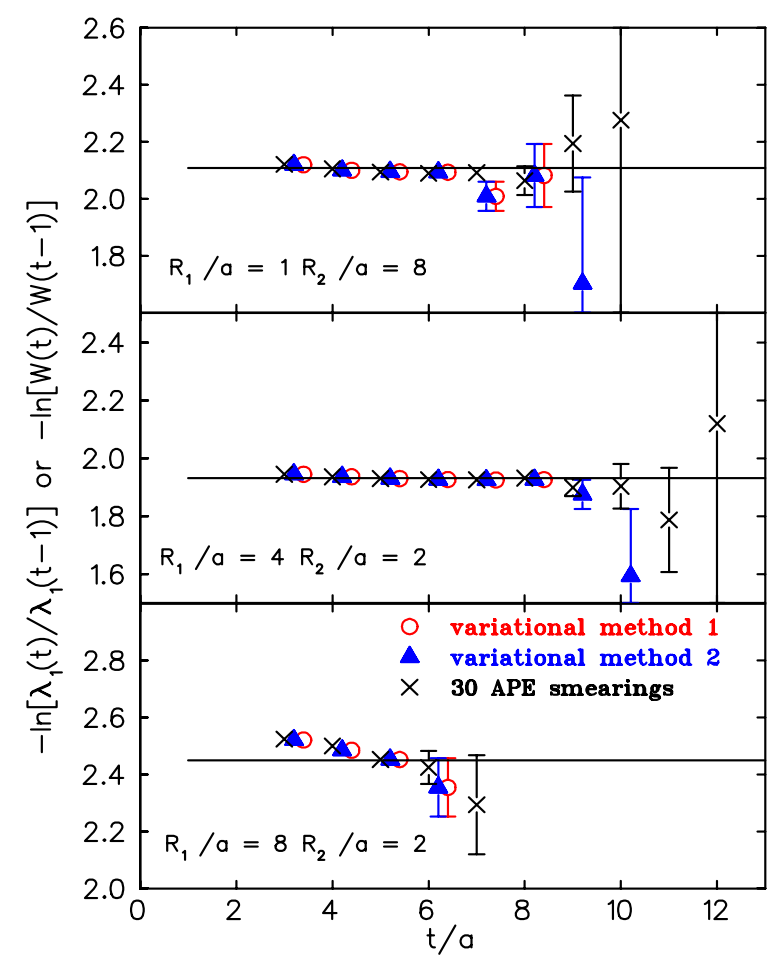

FIG. 14: Plateau values for the pentaquark potential. Top for the case where $R_{1} / a=1$ and $R_{2} / a=8$; middle for $R_{1} / a=4$ and $R_{2} / a=2$; bottom for $R_{1} / a=8$ and $R_{2} / a=2$. The rest of the notation is as in Fig. [6

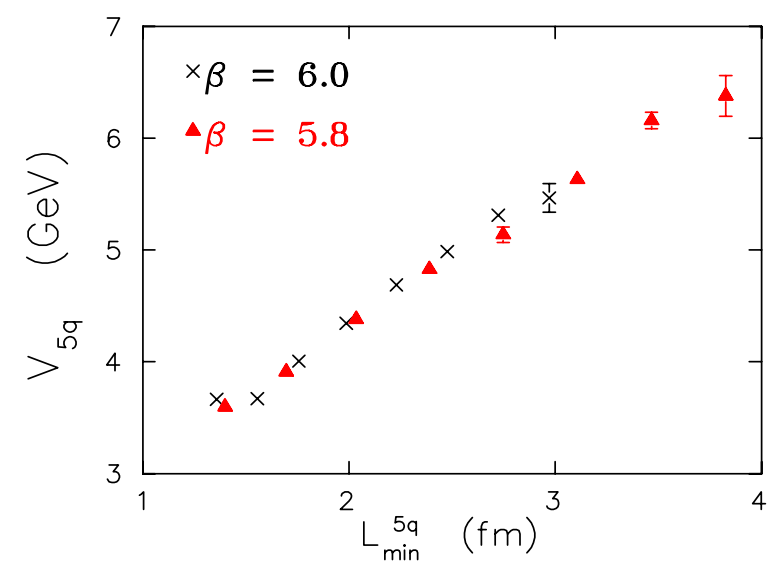

FIG. 15: The pentaquark static potential at $\beta=6.0$ (crosses) and $\beta=5.8$ (filled triangles) for $R_{2}=0.6 \mathrm{fm}$ as a function of the minimal length. The data at $\beta=5.8$ are shifted by a constant.

geometries we have a system of two mesons rather than a genuine four quark bound state. On the other hand when the distance between the diquarks is larger than their internal diquark distance the potential is lower than the sum of the two $q \bar{q}$ potentials. In Fig. 11] we show lattice data for $\beta=5.8$ for the smallest internal diquark separation, namely for $R_{2}=0.3 \mathrm{fm}$, and compare them with $V_{\min }^{4 q}$ and $2 V_{q \bar{q}}\left(R_{1}\right)$. We have chosen $\beta=5.8$ in order to reach larger diquark separations. The tetraquark po-

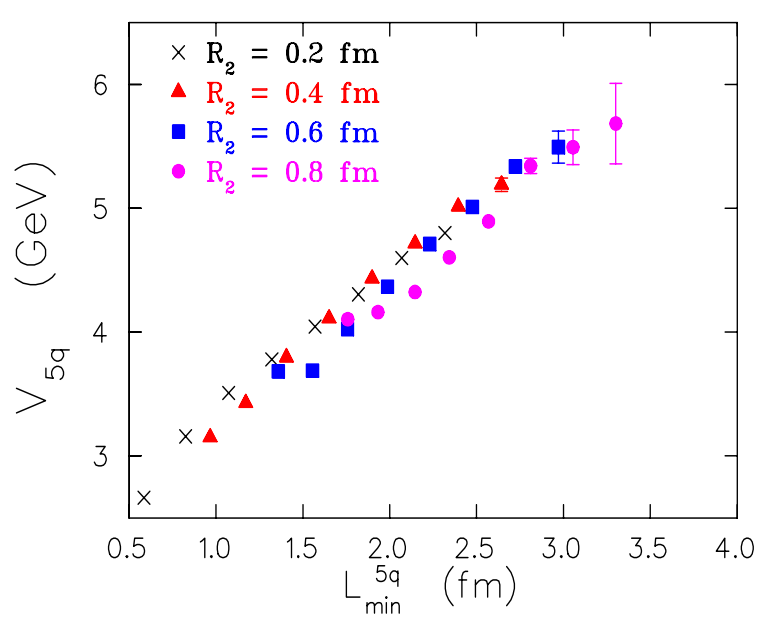

FIG. 16: The pentaquark static potential at $\beta=6.0$ versus the minimal length. The notation is the same as that used in Fig. 9

tential starts as a sum of the two meson potentials and then crosses over to approach $V_{\min }^{4 q}$. Although $V_{\min }^{4 q}$ has a larger slope as compared to the slope of the tetraquark confining potential it approximates best the lattice data for values of $R_{1}$ in the range of about one 0.5 to $1 \mathrm{fm}$. In Fig. 12 we perform the same comparison but for internal diquark distance equal to $0.8 \mathrm{fm}$. In this case the tetraquark potential becomes larger than the sum of the two meson potentials approaching $V_{\text {min }}^{4 q}$ from below. For the two largest distances the data become too noisy and better statistics are required to determine more accurately the long distance behaviour.

We perform a similar analysis for the pentaquark system. The geometry used for the pentaquark potential is shown in Fig. [13] We place the antiquark on the z-axis distance $R_{1}$ from the origin. The two pairs of quarks are placed at distances $\left(R_{1}, 0, \pm R_{2} / 2\right)$ and $\left(0, R_{1}, \pm R_{2} / 2\right)$ so

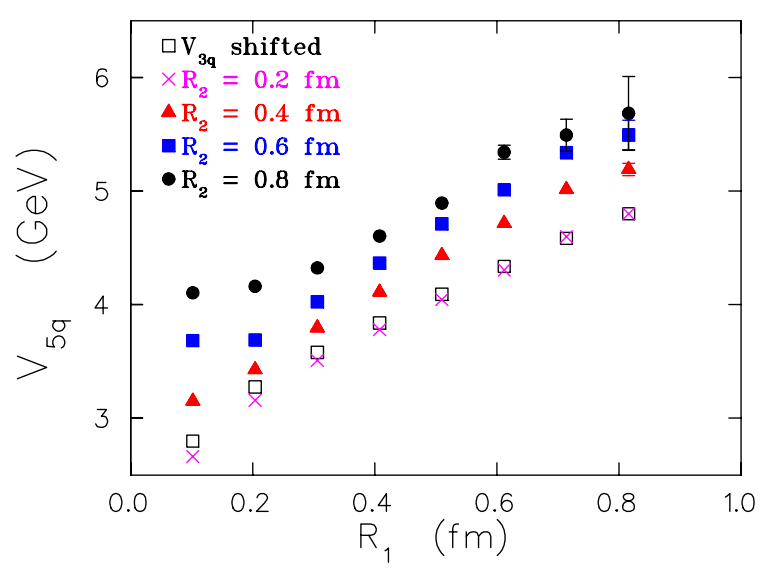

FIG. 17: The pentaquark static potential versus $R_{1}$ for $R_{2}=0.2 \mathrm{fm}$ (crosses), $0.4 \mathrm{fm}$ (filled triangles), $0.6 \mathrm{fm}$ (filled squares) and $0.8 \mathrm{fm}$ (filled circles). The baryonic potential shifted by a constant is shown by the open squares. 
TABLE III: Determination of plateau values for the pentaquark potential at $\beta=6.0$. The notation is the same as that of Table III

\begin{tabular}{|c|c|c|c|c|c|c|c|c|c|c|c|c|c|c|c|c|}
\hline \multicolumn{17}{|c|}{ Pentaquark potential } \\
\hline$R_{1} / a$ & \multicolumn{4}{|c|}{$R_{2} / a=2$} & \multicolumn{4}{|c|}{$R_{2} / a=4$} & \multicolumn{4}{|c|}{$R_{2} / a=6$} & \multicolumn{4}{|c|}{$R_{2} / a=8$} \\
\hline \multirow{4}{*}{1} & $t_{i} / a$ & $t_{f} / a$ & $a V_{5 q}$ & $\tilde{\chi}^{2}$ & $t_{i} / a$ & $t_{f} / a$ & $a V_{5 q}$ & $\tilde{\chi}^{2}$ & $t_{i} / a$ & $t_{f} / a$ & $a V_{5 q}$ & $\tilde{\chi}^{2}$ & $t_{i} / a$ & $t_{f} / a$ & $a V_{5 q}$ & $\tilde{\chi}^{2}$ \\
\hline & 11 & 12 & $1.369(1)$ & 2.8 & 7 & 11 & $1.616(1)$ & 8.5 & 5 & 12 & $1.885(2)$ & 2.9 & 3 & 12 & $2.111(2)$ & 7.5 \\
\hline & 3 & 12 & $1.358(1)^{* a}$ & 0.2 & 9 & 11 & $1.607(3)^{*}$ & 0.4 & 6 & 12 & $1.878(3)^{*}$ & 1.0 & 5 & 12 & $2.093(4)^{*}$ & 0.2 \\
\hline & 5 & 12 & $1.358(2)^{a}$ & 0.2 & 10 & 11 & $1.609(5)$ & 0.5 & 9 & 12 & $1.880(23)$ & 0.9 & 7 & 12 & $2.090(19)$ & 0.2 \\
\hline \multirow{3}{*}{2} & 6 & 11 & $1.620(1)$ & 8.6 & 6 & 11 & $1.760(1)$ & 6.3 & 6 & 12 & $1.926(3)$ & 3.4 & 5 & 12 & $2.122(4)^{*}$ & 1.0 \\
\hline & 9 & 11 & $1.611(2)^{*}$ & 0.1 & 8 & 11 & $1.748(4)^{*}$ & 0.9 & 8 & 12 & $1.881(13)^{*}$ & 0.6 & 6 & 12 & $2.112(7)$ & 0.7 \\
\hline & 10 & 11 & $1.611(4)$ & 0.2 & 9 & 11 & $1.742(7)$ & 0.8 & 9 & 12 & $1.862(32)$ & 0.7 & 8 & 12 & $2.091(60)$ & 0.7 \\
\hline \multirow{3}{*}{3} & 5 & 12 & $1.789(1)^{*}$ & 0.9 & 4 & 11 & $1.960(1)$ & 13 & 5 & 12 & $2.068(3)$ & 2.7 & 4 & 10 & $2.228(3)$ & 9.0 \\
\hline & 8 & 12 & $1.786(4)$ & 0.2 & 7 & 11 & $1.936(5)^{*}$ & 0.1 & 6 & 12 & $2.053(5)^{*}$ & 0.3 & 5 & 10 & $2.205(5)^{*}$ & 1.0 \\
\hline & 10 & 12 & $1.781(20)$ & 0.4 & 9 & 11 & $1.928(37)$ & 0.1 & 7 & 12 & $2.043(11)$ & 0.2 & 6 & 10 & $2.187(11)$ & 0.4 \\
\hline \multirow{3}{*}{4} & 5 & 12 & $1.928(2)^{*}$ & 0.5 & 5 & 8 & $2.105(3)$ & 1.3 & 3 & 11 & $2.258(3)$ & 18 & 3 & 8 & & 25 \\
\hline & 8 & 12 & $1.926(12)$ & 0.4 & 6 & 8 & $2.097(5)^{*}$ & 0.2 & 5 & 11 & $2.227(3)^{*}$ & 1.1 & 5 & 8 & $2.348(7)^{*}$ & 0.5 \\
\hline & 10 & 12 & $1.888(78)$ & 0.2 & 7 & 8 & $2.105(15)$ & 0.1 & 7 & 11 & $2.222(37)$ & 1.3 & 7 & 8 & $2.249(96)$ & 0.1 \\
\hline \multirow{3}{*}{5} & 5 & 11 & $2.063(3)^{*}$ & 0.5 & 3 & 9 & $2.271(3)$ & 5.7 & 4 & 9 & $2.403(4)$ & 1.6 & 4 & 7 & $2.534(6)$ & 4.0 \\
\hline & 7 & 11 & $2.068(11)$ & 0.4 & 4 & 9 & $2.261(3)^{*}$ & 0.6 & 4 & 7 & $2.403(4)^{*}$ & 1.0 & 5 & 7 & $2.496(14)^{*}$ & 0.5 \\
\hline & 8 & 11 & $2.069(36)$ & 0.3 & 7 & 9 & $2.277(40)$ & 0.5 & 5 & 7 & $2.391(8)$ & 0.1 & 6 & 7 & $2.462(62)$ & 0.8 \\
\hline \multirow{3}{*}{6} & 3 & 10 & $2.215(3)$ & 5.6 & 4 & 8 & $2.405(5)^{*}$ & 1.0 & 4 & 10 & $2.556(6)^{*}$ & 1.0 & 3 & 9 & $2.737(6)$ & 3.9 \\
\hline & 5 & 10 & $2.196(6)^{*}$ & 0.5 & 5 & 8 & $2.395(8)$ & 0.6 & 6 & 10 & $2.497(55)$ & 0.4 & 5 & 9 & $2.725(32)^{*}$ & 1.7 \\
\hline & 7 & 10 & $2.192(29)$ & 0.2 & 7 & 8 & $2.322(98)$ & 0.3 & 7 & 10 & $2.492(306)$ & 0.5 & 6 & 9 & $2.590(174)$ & 0.2 \\
\hline \multirow{3}{*}{7} & 4 & 8 & $2.345(6)^{*}$ & 1.0 & 3 & 7 & $2.572(5)$ & 2.6 & 4 & 9 & $2.722(9)^{*}$ & 0.4 & 3 & 8 & $2.904(7)$ & 1.9 \\
\hline & 5 & 8 & $2.333(10)$ & 0.4 & 4 & 7 & $2.558(6)^{*}$ & 1.0 & 5 & 9 & $2.694(29)$ & 0.2 & 5 & 8 & $2.807(72)^{*}$ & 1.2 \\
\hline & 6 & 8 & $2.315(24)$ & 0.3 & 5 & 7 & $2.553(16)$ & 0.1 & 7 & 9 & $2.609(148)$ & 0.1 & 6 & 8 & $2.290(279)$ & 0.1 \\
\hline \multirow{3}{*}{8} & 3 & 7 & $2.513(5)$ & 5.7 & 3 & 9 & $2.724(6)$ & 3.1 & 5 & 8 & $2.807(66)^{*}$ & 1.8 & 5 & 6 & $2.968(165)^{*}$ & 1.1 \\
\hline & 5 & 7 & $2.448(18)^{*}$ & 0.5 & 5 & 9 & $2.648(28)^{*}$ & 1.1 & 6 & 8 & $2.266(245)$ & 0.2 & & & & \\
\hline & 6 & 7 & $2.411(55)$ & 0.5 & 6 & 9 & $2.450(116)$ & 0.1 & 7 & 8 & $2.079(767)$ & 0.4 & & & & \\
\hline
\end{tabular}

${ }^{a}$ This value was extracted using Eq. 18

that when $R_{2}=0$ this configuration reduces to the geometry that we used for the baryonic potential [16]. The quality of the plateaus is seen in Fig. 14 for representa-

In Fig. [15] we compare the results for the pentaquark potential at $\beta=5.8$ and 6.0 for the same physical internal diquark separation $R_{2}$. The data at $\beta=5.8$ are shifted by $5 / 2$ the difference in the values of $V_{0}$ at the two $\beta$ values. As in the case of the tetraquark potential, the results for the pentaquark potential approximately fall on the same curve indicating good scaling. The comments made for the errors on the results obtained at $\beta=5.8$ at the larger distances when discussing the scaling of the tetraquark potential apply also here. Table III gives details of our fitting procedure at $\beta=6.0$ and provides an indication of the systematic errors involved in changing the fitting range.

In Fig. [16] we plot the pentaquark potential evaluated tive values of $R_{1}$ and $R_{2}$. Again the ratio of Wilson loops with the largest number of APE smearings produces the best plateaus.

at $\beta=6.0$ versus $L_{\min }^{5 q}$. Although not as clearly seen as in the case of the tetraquark system there are four sets of curves corresponding to the four different values of $R_{2}$. For large values of $R_{1}$ they converge to the same curve which again suggests that the potential asymptotically depends only on the minimal length at least to a first approximation. The four sets of curves are more clearly seen in Fig. 17 where the pentaquark potential is plotted as a function of the diquark separation $R_{1}$ for the four values of the internal diquark distance $R_{2}=0.2,0.4,0.6$ and $0.8 \mathrm{fm}$. On the same figure we also display our lattice results for the baryonic potential shifted by a constant showing that the linear dependence of the pentaquark potential for small separations $R_{2}$ is approximately the 
same as that of the baryonic potential. It should be noted that, for the geometry used in the evaluation of the pentaquark potential, the sum of the baryonic and mesonic potentials depends on both $R_{1}$ and $R_{2}$ and therefore we can no longer draw a universal curve for all separations $R_{2}$ as we did for the case of the tetraquark potential. Instead we compare the pentaquark potential to $V_{\min }^{5 q}$ and the sum of the baryonic and mesonic potentials in Figs. 18 and 19 for two extreme cases: In Fig. 18 we show results for the smallest possible internal diquark distance, namely for $R_{2}=0.3 \mathrm{fm}$, and consider $\beta=5.8$ so that we can reach larger physical distances in $R_{1}$. In this case the potential approaches $V_{\min }^{5 q}$ for $R_{1}>0.4 \mathrm{fm}$. This means that when $R_{1}$ becomes larger than the internal diquark distance the $q q q q \bar{q}$ system is well described by the minimal flux connecting the quarks. This genuine pentaquark state has static energy which is lower than the sum of the baryonic plus the mesonic potential. In Fig. [19 we show results for the largest possible internal diquark separation, namely for $R_{2}=0.8 \mathrm{fm}$, and consider $\beta=6.0$ since the results for $\beta=5.8$ become too noisy to extract any useful information. For this geometry the quarks in the diquarks are always a distance larger or equal to $R_{1}$. In this case the results are well described by the sum of the baryonic and mesonic potentials and only for distances larger than $0.6 \mathrm{fm}$ they begin to approach $V_{\min }^{5 q}$ from below. More statistics are required to reduce the errors in order to draw a definite conclusion for the large distance behaviour in this case.

\section{CONCLUSIONS}

We have calculated the static tetraquark and pentaquark potentials selecting geometries that are motivated by the diquark picture. Using multi-hit for the time links and APE smearing for the spatial links we obtain results for inter-quark distances of the order of $1 \mathrm{fm}$. Comparing the results obtained at $\beta=5.8$ and 6.0 we show that the potentials have reasonable scaling properties.

The main conclusions regarding the tetraquark potential are: 1) When the two diquarks are closer than $\sim 0.5 \mathrm{fm}$ and with internal diquark distances larger than their separation $\left(R_{2}>R_{1}\right)$ then the $q q \bar{q} \bar{q}$ system breaks, as expected, into two mesons and its static potential is approximately given by the sum of the two meson potentials. The geometry used for the tetraquark potential makes it easy to see when the ground state is a two meson state since then the static potential becomes independent of the internal diquark distance $R_{2}$. 2) For distances between diquarks larger than the internal diquark distance the static potential is approximately described by $V_{\min }^{4 q}$, which has a confining part proportional to the minimal length flux tube joining the quarks. In the parametrization of $V_{\min }^{4 q}$ we used values for $V_{0}, \alpha$ and $\sigma$ extracted from the static $q \bar{q}$ potential and therefore there are no adjustable parameters. The linear dependence of

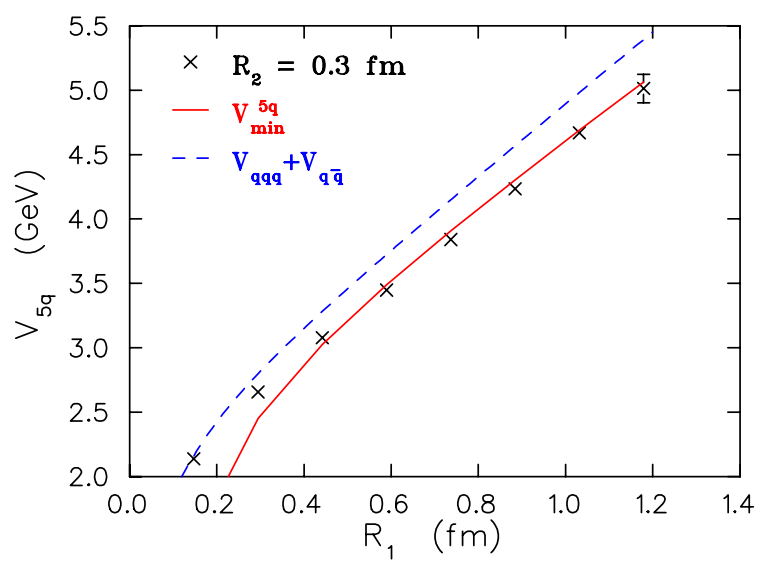

FIG. 18: The pentaquark static potential at $\beta=5.8$ for $R_{2}=0.3 \mathrm{fm}$ compared with $V_{\min }^{5 q}$ (solid line) and with the sum of the baryonic and mesonic potentials (dashed line).

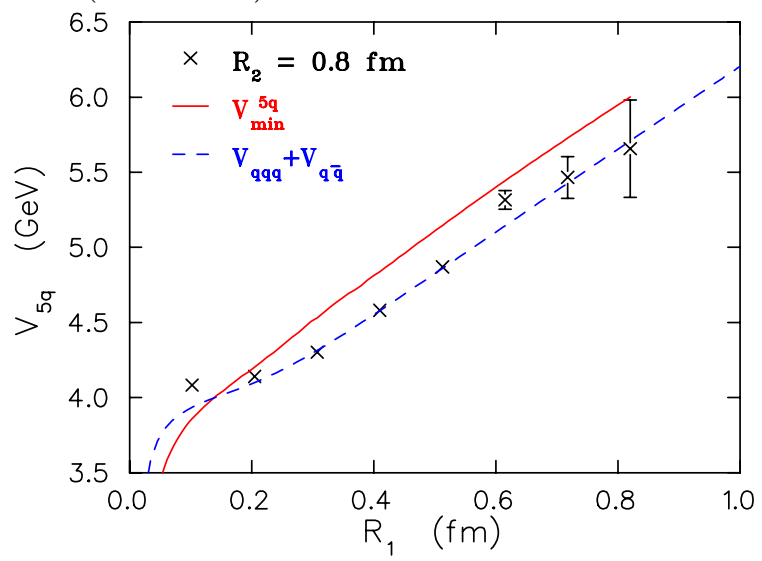

FIG. 19: The pentaquark static potential at $\beta=6.0$ for $R_{2}=0.8 \mathrm{fm}$ compared with $V_{\min }^{5 q}$ (solid line) and with the sum of the baryonic and mesonic potentials (dashed line).

the tetraquark potential would require a smaller value of $\sigma$ as compared to that extracted from the $q \bar{q}$ potential. For internal diquark separations between about 0.5 and $1 \mathrm{fm}$ the potential is larger than the sum of the two meson potentials and approaches $V_{\min }^{4 q}$ from below.

The pentaquark potential shows similar behaviour: For diquark separations $R_{1}$ small as compared to $R_{2}$ the potential is well approximated by the sum of the mesonic and baryonic potentials. For values of $R_{1}$ larger than $R_{2}$ it approaches $V_{\min }^{5 q}$. However in this case $V_{\min }^{5 q}$ provides a better description to the data than it did for the tetraquark case. For our geometry we were able to confirm this behaviour for internal diquark distances $R_{2} \lesssim 0.5 \mathrm{fm}$. For larger values of $R_{2}$ the data indicate that the pentaquark potential also approaches $V_{\min }^{5 q}$ albeit with large statistical errors that need to be reduced before one can make a definite statement. When $R_{2} \lesssim 0.5 \mathrm{fm}$ the pentaquark potential approaches $V_{\text {min }}^{5 q}$ from above. When $R_{2} \gtrsim 0.5 \mathrm{fm}$ the pentaquark poten- 
tial approaches $V_{\min }^{5 q}$ from below at least for distances $R_{1}$ up to $\sim 1 \mathrm{fm}$ studied in this work. Although the exact values for the distances $R_{1}$ and $R_{2}$ where this behaviour is observed may depend on the geometry, the general behaviour should not be affected.

In summary, these observations suggest that, when the separation between diquarks is larger than the internal diquark distances with the latter obtaining values up to about half a fermi, the tetraquark and pentaquark systems behave as multiquark bound states rather than break into mesons or baryons. Although this behaviour is determined using a particular geometry it should hold for general geometries that allow diquark formation.

Acknowledgments: We thank H. Neuberger for encouraging us to look at the static pentaquark potential.

Note Added: After the submission of this work we notice a preprint on the static potential on hep-lat 26].
[1] LEPS collaboration, T. Nakano et al., Phys. Rev. Lett. 91, 012002 (2003); DIANA collaboration, V. V. Barmin et al., Phys. Atom. Nucl. 66, 1715 (2003); CLAS collaboration, S. Stepanyan et al., Phys. Rev. Lett. 91, 252001 (2003); SAPHIR collaboration, J. Barth et al., Phys. Lett. B572, 127 (2003).

[2] D. Diakonov, V. Petrov and M. Polyakov, Z. Phys. A 359, 305 (1997).

[3] F. Csikor, Z. Fodor, S.D. Katz, T.G. Kovacs JHEP 0311, 070 (2003); T.-W. Chiu, T.-H. Hsieh hep-ph/0403020 S. Sasaki, Phys. Rev. Lett. 93 152001(2004).

[4] C. Alexandrou, G. Koutsou and A. Tsapalis, XXII International Symposium on Lattice Field Theory (Lattice 2004), Fermilab, USA, June 21-26, 2004, hep-lat/0409065

[5] N. Mathur et al., Phys. Rev. D 70, 074508 (2004); N. Ishii, et al., hep-lat/0408030

[6] A. Casher and S. Nussinov, Phys. Lett. B578, 124 (2004).

[7] X.-C. Song and S.-L. Zhu, hep-ph/0403093

[8] R. Jaffe and F. Wilczek, Phys. Rev. Lett. 91, 232003 (2003).

[9] R. Jaffe, Phys. Rev. D 15, 267 (1977); C.-K. Chow, Phys. Rev. D 51, 6327 (1995); B. Gelman and S. Nussinov, Phys. Lett. B 551, 296 (2003).

[10] NA49 collaboration, C. Alt et al., Phys. Rev. Lett. 92, 042003 (2004).

[11] G. Parisi, R. Petronzio and F. Rapuano, Phys. Lett. B 128, 418 (1983).

[12] Ph. de Forcrand and C. Roiesnel, Phys. Lett. B 151, 77 (1985).
[13] G.S. Bali, Ch. Schlichter and K. Schilling, Phys. Rev. D 51, 5165 (1995).

[14] APE Collaboration, M. Albanese et al., Phys. Lett. B 192, 163 (1987).

[15] N. A. Campbell, A. Huntley and C. Michael, Nucl Phys. B306, 51 (1988); M. Lüscher and U. Wolff, Nucl. Phys. B339, 222 (1990); M. Guagnelli, R. Sommer and H. Wittig, Nucl. Phys. B535, 389 (1998).

[16] C. Alexandrou, Ph. de Forcrand and A. Tsapalis, Phys. Rev. D 65, 054503 (2002); Nucl. Phys. B (Proc.Suppl.) 106, 403 (2002); Nucl. Phys. (Proc.Suppl.) 109A, 153 (2002).

[17] NERSC archive, G. Kilcup et al., hep-lat/9609006

[18] J. Carlson, J. Kogut and V. R. Pandharipande, Phys. Rev. D 27, 233 (1983).

[19] J. M. Cornwall, Phys. Rev. D 54, 6527 (1996).

[20] J. M. Cornwall, Phys. Rev. D 69, 065013 (2004).

[21] R.Sommer and J.Wosiek, Phys. Lett. B 149, 497 (1984); Nucl. Phys. B267, 531 (1986).

[22] G. S. Bali, Phys. Rep. 343, 1 (2001).

[23] C. Alexandrou, Ph. de Forcrand and O. Jahn, Nucl. Phys. B (Proc.Suppl.) 119, 667 (2003).

[24] T. T. Takahashi, H. Matsufuru, Y. Nemoto and H. Suganuma, Phys. Rev. Lett. 86, 18 (2001); T. T. Takahashi, H. Suganuma, Y. Nemoto and H. Matsufuru, Phys. Rev. D 65, 114509 (2002).

[25] H.Ichie et al., Nucl. Phys. A721, 899 (2003); V. G. Bornyakov et. al, hep-lat/0401026

[26] F. Okiharu, H. Suganuma and T. T. Takahashi, hep-lat/0407001 